University of Nebraska - Lincoln

DigitalCommons@University of Nebraska - Lincoln

20th \& 21st Century French and Francophone Modern Languages and Literatures, Department Studies International Colloquium

\title{
Ce que le mirage doit à l'oasis de Yasmina Khadra: Comment devient-on enfant du Sahara?
}

Brigitte Tsobgny

Follow this and additional works at: https://digitalcommons.unl.edu/ffsc2020

Part of the Comparative Literature Commons, French and Francophone Literature Commons, and the Other French and Francophone Language and Literature Commons

This Article is brought to you for free and open access by the Modern Languages and Literatures, Department of at DigitalCommons@University of Nebraska - Lincoln. It has been accepted for inclusion in 20th \& 21st Century

French and Francophone Studies International Colloquium by an authorized administrator of DigitalCommons@University of Nebraska - Lincoln. 


\title{
Ce que le mirage doit à l'oasis de Yasmina Khadra Comment devient-on enfant du Sahara?
}

\author{
Brigitte Tsobgny, $\mathrm{PhD}$ candidate \\ Department of Modern Languages and Linguistics \\ Florida State University
}

\section{Résumé}

Être un enfant du Sahara, est-ce seulement être capable d'escalader pieds nus les barkhanes brûlantes? Dans Ce que le mirage doit à l'oasis, Yasmina Khadra invite le lecteur à découvrir son Sahara natal à travers différents personnages. Pour les uns, le Sahara c'est l'ennui, le silence, l'immobilité. Pour d'autres, c'est un lieu d'inspiration, de méditation, de prières ou d'apaisement. Pour d'autres encore, c'est un terrain vague juste bon pour les courses automobiles ou des essais nucléaires. Les narcissiques y voient une terre de conquête, les jihadistes ont fait de ses grottes des repaires d'assassins. La culture ou l'époque peuvent-elle expliquer ces différences ? Peut-on les attribuer à une interaction forte entre l'homme et la terre? Ce sont les questions auxquelles je vais répondre dans cette présentation en analysant les rapports au corps, au temps et à l'environnement des personnages de Ce que le mirage doit à l'oasis, nés ou de passage au Sahara. Cela revient dans un premier temps à montrer comment le désert agit sur ses enfants naturels, dans un deuxième temps comment il pénètre dans l'esprit et le corps des enfants venus d'ailleurs, les transforme avant de les adopter. 
Dans Ce que le mirage doit à l'oasis, Yasmina Khadra invite le lecteur à découvrir son Sahara natal ainsi que ses enfants naturels et adoptifs. En effet, parmi les randonneurs, explorateurs, pèlerins, aventuriers ou colons qui l'ont visité ou qui s'y sont établis, le Sahara a adopté des enfants venus d'ailleurs dont Charles de Foucauld, le père alsacien pour qui le désert a été un lieu de révélation et Isabelle Eberhart, la Suissesse « Rimbaud au féminin » qui y allait souvent pour se ressourcer.

Quelles sont les caractéristiques des enfants du Sahara selon le texte de Yasmina Khadra? C'est la question à laquelle je vais tenter de répondre dans cette présentation en analysant les rapports au corps, au temps, à la société et à l'environnement des personnages de Ce que le mirage doit à l'oasis. Cela revient à montrer dans un premier temps, comment le désert façonne ses enfants naturels, puis dans un deuxième temps comment il pénètre dans l'esprit et le corps des enfants venus d'ailleurs, comment il les transforme avant de les adopter. Mais avant, il serait intéressant de considérer la terre elle-même, la terre qui porte ces enfants.

\section{Le Sahara de Yasmina Khadra}

Ce que le mirage doit à l'oasis est une autofiction, une conversation entre le désert et le narrateur qui n'est autre que Yasmina Khadra lui-même, né à Kenadsa, dans le sud-Algérien en 1955 (p. 12). «Toute chose en ce monde a une fin» (p.13), ce sont les premiers mots que Khadra attribue au désert dans ce texte très lyrique où le Sahara dresse son autoportrait. Personnage à part entière, le désert se présente sous un ton nostalgique comme une terre du passé :

Mes forêts se ramifiaient à perte de vue, frémissantes de fraicheur, peuplées de fauve gigantesques et de rapaces grands comme des vaisseaux spatiaux. Je naissais au jour dans le coup de gueule des volcans et m'assoupissais le soir dans le clapotis des 
cascades. (...) Je me croyais éternel, sauvage et indomptable, aussi redoutable que mes plantes carnivores, aussi imprenable que le bruissement de mes taillis... (p. 14).

Comment habiter un espace au passé florissant mais dont les certitudes ont été balayées ? Avec un ton modeste, le Sahara invite forcément ses enfants à faire preuve d'humilité. « Et regardez ce que le Temps a fait de moi : un désert ! (p. 14). Face au temps, avec un T majuscule, le désert admet sa défaite ; le temps lui a confisqué ses fleuves, ses lacs, ses jungles, tout ce qui faisait sa grandeur. Le Sahara n'est plus qu'un paradis perdu, « pauvre, misérable et nu » (p. 14) qui considère qu'il n'a plus grand-chose à offrir. Ce à quoi le narrateur, humain qui vit dans un corps périssable, lui répond : «C'est dans ton mutisme que j'ai entendu chanter mon cœur d'enfant. Si je suis devenu l'homme que je suis, si j'ai choisi d'aimer les êtres et les choses, c'est grâce à toi » (p. 29/30). Pour le narrateur, les richesses du Sahara sont inestimables. Bien entendu, il ne s'agit pas de richesses matérielles puisque le temps lui a tout volé, c'est justement son silence, sa pauvreté que Khadra considère comme source de bienfaits. C'est cette pauvreté qui a façonné l'homme qu'il est devenu.

Si tes fleuves se sont tus, si tes lacs ont disparu, c'est pour que tu fasses peau neuve. Tu renais au temps des ascèses (...) il est inconcevable de déplorer le songe quand on est la beauté, de renoncer à l'espoir quand on a survécu aux cataclysmes, de résilier les promesses quand rien n'est tout à fait perdu (p. 19).

Mieux que le Sahara lui-même, ses enfants connaissent ses trésors. Être enfant du désert est-ce mener une vie d'ascète ? Qu'y a de si beau dans les étendues d'ergs ${ }^{1}$ taciturnes, de regs ${ }^{2}$ brûlants ou de barkhanes immobiles ? Comment le désert agit-il sur ses enfants naturels ? Quels sont leurs rapports au corps et au monde environnant?

\footnotetext{
${ }^{1}$ Erg : désert de sable.

${ }^{2}$ Reg : désert de pierres.
} 


\section{Comment le désert agit-il sur ses enfants naturels?}

Selon Yvan Amar, notre rapport au corps et notre rapport au monde sont profondément imbriqués. Le rapport au corps est à la fois une donnée biologique, psychologique, sociale et spirituelle et par conséquent, il est conditionné socialement et culturellement (Amar : 2005, 157). Dans le même esprit, Descola nous invite à « rendre toute son importance au corps comme instrument de connaissance » tout en saisissant les «structures de cadrage qui rendent compte de la cohérence et de la régularité des comportements des membres d'une communauté » (Descola dans Boëtsch : 2007, 5- 6). Analyser le rapport au corps des enfants naturels du Sahara peut aider à comprendre leurs relations avec le Sahara, leurs manières d'appréhender le monde.

Un des passages les plus parlants sur le corps dans le récit de Khadra, c'est quand le narrateur, après un long séjour à Oran, revient dans l'oasis où il est né. Ses cousins lui demandent de prouver qu'il est « toujours l'enfant du Sahara » (p.111), un vrai fils du Sahara devant être capable d'escalader pieds nus une dune brûlante sous un soleil de plomb, défi que le narrateur accepte de relever. « J'ai failli prendre feu en atteignant le sommet de la barkhane, mais j'ai mérité mon statut de Bédouin à part entière, ce jour-là » (p. 112). Être un fils du Sahara, c'est donc être capable de s'accommoder des rigueurs climatiques de l'environnement. Mais Khadra ne s'arrête pas là. Les personnages de Ce que le mirage doit à l'oasis, membres de la famille ou de la tribu du narrateur, se contentent de pas grand -chose pour nourrir leur corps. Après la déroute de sa tribu «qui a défendu son territoire bec et ongles contre l'invasion française » (p. 163), son grand-père s’isole dans le désert pour écrire et méditer. « Il restait des semaines absent, subsistant de dattes et d'eau aromatisée à l'huile de cade » (p. 163). Et quand les Bédouins ne se livrent pas à une vie ascétique, ils ne reculent pas devant l'effort et soumettent volontiers leurs corps aux risques les plus fous. A quatorze ans, après la dislocation de la tribu vaincue par l'armée coloniale, celui qui deviendra le père du narrateur part à la recherche de ses frères disséminés dans le Sahara ; «il ira jusqu'au Maroc, à pied, sans un sou 
et sans boussole, et évitera par miracle d'être égorgé par des brigands du Rif » (p. 49-50). S'aventurer ainsi dans l'inconnu, sous des températures extrêmes, au mépris de la soif, de la faim et de l'insécurité relève certes de l'inconscience, mais ce qui motive cet acte fou, c'est le désir du père du narrateur de rendre le sourire à son père à lui. S'aventurer ainsi dans l'inconnu, c'est aussi se moquer du temps qui passe, seul ce qui compte, c'est l'objectif visé. Des années plus tard, il rentrera dans son oasis natale avec deux de ses frères. On l'aura bien compris, le rapport au temps des enfants du Sahara où le temps semble immobile n'est pas le même que celui des cultures occidentales. D'autre part, pour se risquer à un pari aussi hasardeux, il faut croire à l'invraisemblable. Le Sahara est un lieu de mystères, il trompe les sens de ses enfants et de ses visiteurs qui, par conséquent, ont tendance à croire à l'invraisemblable. Par exemple, le narrateur, alors capitaine de l'armée algérienne, s'apprête à camper une nuit avec sa troupe dans le désert du Ténéré, « le terrible désert des déserts » (p. 67). Dans un silence crépusculaire, le groupe entend soudain des sons de tambourins et des youyous. Seulement, le village le plus proche se trouve à trois cents kilomètres. Pour calmer ses soldats qui redoutent la présence des djinn $^{3}$, le capitaine monte au sommet d'une crête et scrute les alentours pour identifier l'origine de la clameur festive. Il n'y a rien, « rien que le roulement surnaturel d'une fête impossible à situer » (p. 67). Plusieurs hypothèses peuvent expliquer ce phénomène : une hallucination collective ou plus vraisemblablement «le chant des dunes » résultant des vibrations du sable est en mouvement (Dagois-Bohi : 2010, 9). Quoiqu'il en soit, pour les enfants du désert, c'est un mystère parmi les innombrables mystères que recèle le Sahara : «j'ai appris à croire en l'invraisemblable et en l'absurde puisque dans le désert, ce qui est impensable se réalise pleinement sous nos yeux incrédules » (p. 74), admet le narrateur.

Habiter le Sahara, ce n'est pas seulement se soumettre aux lois des températures extrêmes, du vent, du sable et d'autres éléments de la nature. C'est aussi trouver son bonheur

\footnotetext{
${ }^{3}$ Djinn : démon hostile à l'homme.
} 
dans des plaisirs simples de la vie, comme le témoignent ces paroles du narrateur : «Il me suffisait de m'arrêter au pied de tes pains de sucre pour que le danger s'évanouisse, de siroter un thé à l'ombre d'un rocher-cathédrale pour accéder à l'ivresse de toutes les fêtes » (p. 78). Le bonheur se vit également dans la simplicité de la vie familiale, avoir un bon époux (ou une bonne épouse) sans d'autres prétentions, avoir des enfants. Les parents du narrateur ne s'étaient jamais rencontrés avant la nuit de leurs noces (p. 39). Ce fut pourtant un mariage d'amour (p. 54). «Je le croyais louchon, il me croyait chauve » (p. 39), lui confiera sa mère. Une fois le mariage consommé, le jeune marié voulait un héritier, même s'il n'avait pas grand-chose à lui léguer. «Il voulait un prince, et tant pis s'il était pieds nus » (p.45). Les princes aux pieds nus prolifèrent dans le Sahara, car les enfants du désert, loin de se plaindre des conditions de vie extrêmes, se réjouissent des richesses immatérielles qui abondent dans leur terre. Par exemple, la musique dans le désert «pénètre la pierre et la chair comme une seconde âme » (p. 134). Le désert est également un lieu d'inspiration, un lieu propice à l'écriture, un lieu de contemplation qui rapproche l'homme de lui-même. Belle déclaration d'amour que celle que le narrateur fait à sa terre :

Je te dois l'audace de mes mutineries, les laves dans mes veines ancrées dans tes volcans; je te dois la force tranquille de mes muses et mes fulgurances s'inspirant de tes étoiles filantes ; (...) je te dois mes lettres d'amour aux égéries mortes au large des interdits ; je te dois chaque mot qui me traverse l'esprit (...) (p. 78-79).

Si le désert est un lieu d'inspiration, c'est aussi un lieu qui invite à la sagesse. Dans cette conversation qu'il mène avec le narrateur, il reconnait que les peuples qui l'ont habité comprenaient sa maxime selon laquelle «toute chose a une fin ». Le désert ajoute : «Puisque rien n'est acquis pour toujours, pourquoi le vouloir coûte que coûte ? Les gens du Sahara étaient sages. Ils s'inspiraient de mes nudités pour habiller leur âme et trouvaient dans mes silences de quoi nourrir leur esprit. Ils étaient riches de leur pauvreté, repus de leur frugalité (...) » (p. 165). 
Comme le souligne D’Andréa, habiter un lieu, c'est « établir avec lui une relation » (D’Andréa, 2017), c'est s'en inspirer aussi pour se construire. La pauvreté serait donc un moteur de création pour les enfants du désert qui ont conscience de la finitude de toute chose et qui savent s'accommoder des rigueurs de la nature qui fourmille de trésors si l'on sait bien la regarder. Pour être capable de jouir de ces trésors, faut-il être né dans le Sahara? De tous temps, ce désert a fasciné des hommes et des femmes. Le Sahara a beaucoup d'enfants adoptifs. Ont-ils des dénominateurs communs ? Le désert les transforme-t-il avant de les adopter ?

\section{Caractéristiques des enfants adoptifs du désert}

De même que le Sahara de Khadra renie certains de ses enfants naturels comme les jihadistes qui «troublent [s]es ascèses à coups de prêches assassins et de carnages ignobles » (p. 121), il n'adopte pas tous ceux qui foulent son sol. Faisant sans doute allusion aux participants du rallye Paris-Dakar, il regarde d'un œil désapprobateur ceux qui sur les bécanes et les camions, «soulèvent [s]a poussière comme un trophée et foncent sur [s]es pistes en conquérants » (p. 120), alors qu'il n'aspire qu'à être « un havre de paix et de recueillement » (p. 121). Il en veut également aux archéologues qui profanent ses tombeaux et pillent ses patrimoines (p. 121). Il apprécie peu ces visiteurs qui «n'ont d'yeux que pour euxmêmes » (p.121) et qui ne viennent que pour s'y prendre en photos : «ils passeraient devant la chance de leur vie qu'ils ne la verraient pas » (p. 121), regrette-t-il. Il éprouve encore moins de sympathie pour ceux qui y ont étendu du béton, ceux qui ont pollué son sol ainsi que ceux qui le prennent pour un terrain vague, une espèce de no man's land, où on peut faire toutes sortes d'abominations, évoquant entre autres les essais nucléaires effectués par la France avant l'indépendance de l'Algérie (p. 101). De toute façon, ceux qui ne respectent pas le désert n'ont aucunement l'intention d'y élire domicile, ils ne sont que de passage et repartent comme ils sont arrivés : «Tu ne peux pas savoir combien je plains ces gens qui viennent jusqu’à moi pour 
repartir bredouilles, qui ne verront pas mes miracles ni n'entendront mes hatif ${ }^{4} \ldots »$ (p. 122), déplore le désert.

Les enfants que le Sahara adopte ont des manières de vivre bien différente. Isabelle Eberhart, la poétesse d'origine suisse, en fait partie. Pas seulement parce qu'elle aimait cette terre ; « nos gens l'aimaient bien » (p. 108), précise le narrateur, ce qui sous-entend qu'elle les aimait en retour. Dans ce cas, l'adoption est réciproque, en même temps que Isabelle Eberhart adopte la terre et ses habitants, le Sahara et ses habitants l'adoptent à leur tour. C'est dans sa terre d'adoption qu'elle a trouvé la mort en 1904 alors qu'elle n'avait que 27 ans (p. 109).

Charles de Foucauld est également un fils adoptif du Sahara. Avant de fouler ce qui deviendra sa nouvelle terre, c'était un chrétien sceptique (p. 117) qui « visitait des bordels, prenait des cuites et avait des envies suicidaires » p 118, « il cherchait désespérément quelque chose sans savoir ce que c'était» (p. 119). Et c'est dans le Sahara de Khadra qu'il a eu sa révélation :

Et puis, un soir à l'heure où le soleil saigne sur le basalte de mes montagnes, dans le silence sidéral de mes nudités, (...) l'enfant de Strasbourg eut une révélation : il rencontra son Seigneur, là, dans mon Sahara, au milieu de nulle part qui est aussi le nombril du monde. (...) Il fallait le voir, en larmes et en ferveur, le visage soudain radieux malgré la brûlure des fournaises (...) (p. 119).

Si d'un côté, le désert est un « havre de paix et de recueillement, une aubaine inestimable pour celui qui veut renaître à la beauté des choses, à l'amour et à la fraternité »(p.121), un lieu de révélation, ses enfants adoptifs doivent s'adapter à ses rigueurs climatiques pour profiter de ces bienfaits. Et comme pour montrer une fois de plus que les étrangers adoptés par le Sahara étaient

\footnotetext{
${ }^{4}$ hatif : appel du désert, voix sans corps.
} 
acceptés à la fois par la terre et par les Hommes, le narrateur ajoute : «L'Algérie se souvient encore du père alsacien. (...) Ma tribu a accueilli le père Foucauld en 1884. Il s'est invité à Kenadsa en ami et les miens l'ont reçu en frère » (p. 125). Cette confiance mutuelle passe par le respect des valeurs et des cultures des uns et des autres. Charles de Foucauld respectait les habitants du Sahara, leurs religions ainsi que leurs cultures et, même s'il tenta plusieurs fois de les convertir au christianisme, il était « convaincu que ce qu'il avait à dire, nos Anciens se l'étaient dit depuis très très longtemps » (p. 127) tient à préciser le narrateur. Comme Isabelle Eberhart, c'est dans le désert, à Tamanrasset que Charles de Foucauld trouve la mort en 1916, assassiné.

Khadra donne un autre exemple d'un étranger en voie d'adoption par le désert dans une petite fiction écrite par le narrateur et intitulé Gomri et le capitaine. S'inspirant des batailles que mena sa tribu contre l'armée coloniale, il imagine la vie d'un capitaine de l'armée française envoyé dans le Sahara, non sans avoir précisé auparavant qu'après avoir conquis l'Algérie en 1830, la France a mis soixante-treize ans pour venir à bout des guerriers de sa tribu (p. 81). Le capitaine Ventrabène n'est pas encore adopté par le désert, d'où l'intérêt de la fiction, car elle montre les mécanismes de l'adoption. Dans un lieu perdu du Sahara, le capitaine français se retrouve avec son serviteur arabe, un va-nu-pieds, un «vieillard déshydraté, aussi abîmé et impénétrable qu'une momie » (p. 89). Au début de son séjour, il ne voit dans le désert que «l'ennui, le silence, la nudité » (p. 84). Au bout de trois mois, il a le sentiment que « le sable pénètr[e] ses pores, s'infiltr[e] dans ses veines, assèch[e] son sang et ciment[e] ses pensées (...)» (p. 84). Il y a une mutation qui s'opère à la fois dans son corps et son esprit, car « [n]otre rapport au corps est d'emblée et en quelque sorte spiritualisé »Caron, 2008). A force de s'ennuyer, de retourner les mêmes idées dans la tête, de ne voir en le désert que des étendues immobiles, à force d'avoir l'impression qu'il vit sur une nature morte, le capitaine s'aperçoit qu'il perd « ses repères et ses certitudes : il était en train de s'effranger, de s'émietter, de tomber 
en poussière » (p. 85). Le désert semble l'interroger à le rendre fou : «Il était partout, à perte de vue, à perte de raison, déployé telle une farce incontournable. Les dieux eux-mêmes s’y sentiraient pris en otages » (p. 86). A part les mouches contre qui il se bat et le vieil arabe qu'il considère à peine, il n'y a quasiment pas de vie autour de lui. Les promenades qu'il fait autour de sa maison comme un fauve en cage ne suffisent pas à lui calmer les nerfs. De son ennui, naît le doute qui va s'avérer salvateur ; par exemple, il croit apercevoir un rapace, mais n'est pas sûr de ce qu'il voit, comme si ses sens le trompaient : «C'était aussi cela, le désert, pour quelqu'un qui débarquait de la métropole : n'être plus sûr de rien » (p. 92). Si l'immobilité et l'ennui lui donne des idées suicidaires, il va se ressaisir très vite, car ce n'est pas un homme à capituler aussi facilement. «Ce n'est pas cette saloperie de désert qui va me mettre à genoux » (p. 88), s'insurge-t-il. Finalement, c'est parce qu'il s'ennuie dans le silence et la nudité du désert qu'il s'aperçoit véritablement de la présence de son serviteur arabe, «l'une des rares traces de vie que lui concède le désert » (p. 89), un homme à qui il n'aurait prêté aucune attention en d'autres circonstances. Son serviteur arabe, invisible au départ, finit par apparaître à ses yeux comme un homme ; et même s'il le trouve insaisissable et mystérieux, il réalise surtout que c'est un homme. Tout se passe comme si dans le désert, toute hiérarchisation disparaissait. Le narrateur n'achève pas son manuscrit et ne va pas jusqu'à la mue complète du capitaine, mais ce qui est dévoilé montre que sa transformation s'opère à la fois à travers son corps et son esprit, et ce, grâce à sa persévérance.

\section{Conclusion}

Selon Khadra, les enfants du Sahara ont une interaction forte avec leur terre, qu'ils soient enfants naturels ou adoptifs, quelle que soit leur culture d'origine et leur époque. C'est parce qu'ils acceptent de soumettre leur corps aux rigueurs climatiques et leur esprit à ce qui paraît comme de l'immobilité que des liens forts s'établissent avec leur environnement. L'ennui, le 
silence et la nudité ne sont finalement que les points d'une ligne de départ qui mène vers les richesses inestimables. Ces richesses sont immatérielles. Il faut préciser que le Sahara n'est pas qu'une terre, c'est aussi ses hommes et ses femmes.

Ce que le mirage doit à l'oasis, Yasmina Khadra, Flammarion, 2017.

\section{Works cited}

Amar, Y. (2005). L'effort et la grâce. Paris : Albin Michel.

Boëtsch, Gilles. «Entretien avec Philippe Descola », Corps, vol. 3, no. 2, 2007, pp. 5-11.

Caron, Maxence. «Sur la question du corps dans la pensée de Heidegger. De Sein und Zeit aux Séminaires de Zollikon », Archives de Philosophie, vol. tome 71, no. 2, 2008, pp. 309-329.

D'Andrea, Fabio. «Imago mundi. L'expérience du corps dans le monde », Corps, vol. 15, no. 1, 2017, pp. 89-100.

Dagois-Bohy, Simon. Le Chant des Dunes, Mouvements Collectifs dans un Écoulement Granulaire. Acoustique [physics. class-ph]. Université Paris-Diderot - Paris VII, 2010. Français. tel-00770253. Thèse pour obtenir le titre de Docteur en Sciences de 1'Université Paris Diderot (Paris 7) Mention : Physique.

https://tel.archives-ouvertes.fr/file/index/docid/770253/filename/Dagois-bohy Chant des Dunes.pdf 